\title{
CONGRUÊNCIA E ALGUMAS APLICAÇÕES
}

Richard Mariano de Souza Silva, Julia Rafaela Otero Gonçalves, Fernando Pereira de Souza, Eugenia Brunilda Opazo Uribe.

Universidade Federal de Mato Grosso do Sul, Grupo PET Conexões de Saberes - Matemática, Três Lagoas, MS.E-mail: richard.mariano.mat@gmail.com

Agência de fomento: Programa de Educação Tutorial

\section{RESUMO}

O presente artigo é resultado de um trabalho de pesquisa de alunos do Curso de Matemática no âmbito do Programa de Educação Tutorial, desenvolvido com o objetivo de explorar aspectos teóricos e aplicações do cotidiano de congruências. O trabalho foi desenvolvido em grupo através de pesquisa bibliográfica e estudo teórico, incluindo seminários, exercícios e interpretações de problemas rotineiros, nos quais pode se encontrar congruências em suas aplicações. Será feita uma breve introdução sobre congruências e divisibilidade, e em seguida, algumas aplicações do mesmo, como por exemplo, verificação dos dois últimos dígitos do CPF de uma pessoa, o ultimo digito do ISBN, entre outras aplicações.

Palavras-chave: Divisibilidade, Congruências, Aplicações, Sistemas de Identificação, Programa de Educação Tutorial.

\section{CONGRUENCE AND SOME APLICATIONS}

\begin{abstract}
The present article is the result of a research paper of students from the Mathematics Graduation in the Tutorial Educational Program, developed with the main goal to explore theoretical aspects and daily applications of congruences. The paper was developed by the group through bibliographic research and theoretical study, including seminars, exercises and interpretations of daily problems, in which are found congruences in their applications. A brief introduction on congruences and divisibility will be made and afterwards, some applications of the same, such as verification of the last two digits of a person's CPF, the last digit of ISBN, among other applications.
\end{abstract}

Keywords: Divisibility, Congruence, Applications, Identification Systems, Tutorial Education Program. 


\section{INTRODUÇÃO}

A congruência (ou congruência módulo $\mathrm{m}$ ) é uma importante ferramenta da Teoria Elementar dos Números com diversas aplicações. O matemático alemão Carl Friedrich Gauss (1777 - 1855) colaborou muito com o seu desenvolvimento, segundo Sá (2007), foi Gauss quem observou o uso sucessivo da frase " $a$ dá o mesmo resto que $b$ quando divididos por $m$ " e inclusive, introduziu uma notação especifica para isto, e a denominou "congruência".

O presente trabalho tem como objetivo apresentar os resultados de um estudo teórico sobre o tema em busca de algumas aplicações cotidianas para esse conceito.

\section{METODOLOGIA}

O trabalho foi desenvolvido em grupo através de pesquisa bibliográfica e um estudo teórico que incluiu apresentação de seminários e resolução de exercícios.

\section{RESULTADOS}

Ao estudarmos o conjunto dos números inteiros nos deparamos com o problema da divisão euclidiana ou divisão com restos, que é introduzido no estudo da Matemática desde o ensino fundamental. Iniciaremos o trabalho apresentando o conceito de divisibilidade, o algoritmo da divisão, bem como a definição de congruência e algumas propriedades básicas, que aceitaremos sem demonstração, pois o nosso objetivo é apresentar algumas aplicações, escolhidas entre problemas matemáticos e problemas do cotidiano.

Definição 1. Dados $a, b \in \mathbb{Z}$, com $b \neq 0$, diremos que $b$ divide $a$ se, e somente se, existe um número $c \in \mathbb{Z}$ tal que $a=b \cdot c$.

Proposição 1. (Algoritmo da Divisão) Sejam $a, b \in \mathbb{Z}$, com $a>0$, então existem únicos, $r, q \in \mathbb{Z}$, tais que $a=b \cdot q+r$, com $0 \leq r<a$.

Definição 2. Sejam $a, b, m \in \mathbb{Z} \operatorname{com} m>1$, diremos que $a$ é congruente a $b$ modulo $m$, se $a$ e $b$ possuírem o mesmo resto ao serem divididos por $\mathrm{m}$, e denotaremos $a \equiv b(\bmod m)$.

Exemplo 1: $27 \equiv 31(\bmod 4)$, pois tanto 27 quanto 31 deixam resto 3 na divisão por 4 .

Proposição 2. Dados $a, b, m \in \mathbb{Z} \operatorname{com} m>1$, temos que $a \equiv b(\bmod m)$ se, e somente se, $m \mid(b-a)$.

Proposição 3. Sejam $a, b, c, d, m, r$ números inteiros, com $m>1$ e $r \geq 1$. Então:

I. $\quad a \equiv a(\bmod m)$.

II. Se $a \equiv b(\bmod m)$, então $b \equiv a(\bmod m)$.

III. Se $a \equiv b(\bmod m)$ e $b \equiv c(\bmod m)$, então $a \equiv c(\bmod m)$.

IV. Se $a \equiv b(\bmod m)$ e $c \equiv d(\bmod m)$, então $a+c \equiv b+d(\bmod m)$.

V. Se $a \equiv b(\bmod m)$ e $c \equiv d(\bmod m)$, então $a \cdot c \equiv b \cdot d(\bmod m)$.

VI. Se $a \equiv b(\bmod m)$, então $a^{r} \equiv b^{r}(\bmod m)$.

VII. $\quad a+c \equiv b+c(\bmod m)$ se, e somente, se $a \equiv b(\bmod m)$.

VIII. Se $a b \equiv a c(\bmod m)$ e $\operatorname{md} c(a, m)=1$, então $b \equiv c(\bmod m)$.

Apresentaremos três exemplos de problemas matemáticos que podem ser resolvidos facilmente utilizando o conceito de congruências e apresentaremos também três aplicações do conceito de congruência em problemas do cotidiano.

Exemplo 2: Retirado do Banco de Questões da OBMEP 2010 (Olimpíada Brasileira de Matemática das Escolas Públicas).

Em que fio? A, B, C, D, E, F, G e H são os fios de apoio que uma aranha usa para construir sua teia conforme mostra a figura 1. A aranha continua seu trabalho. Sobre qual fio de apoio estará o número 118 ?

Figura 1. Teia de aranha numérica 


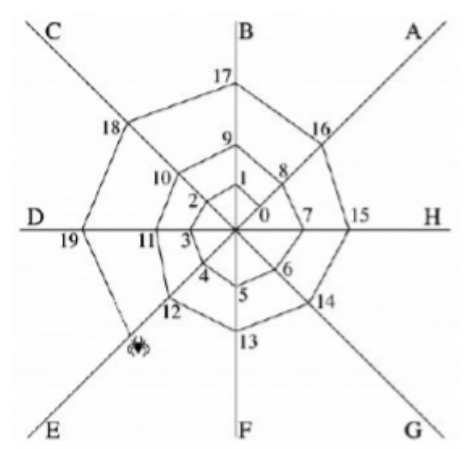

Fonte: Banco de Questões OBMEP 2010.

Solução do exemplo 2: Observando a figura 1, vemos que, sobre o fio A se encontram os múltiplos de 8 , isto é, os números que deixam resto zero quando divididos por 8 e podem ser representados simbolicamente por $8 \mathrm{n}$, com $n \in \mathbb{N}$; sobre o fio $\mathrm{B}$ aparecem os (múltiplos de 8 )+1, isto é, números que podem ser representados simbolicamente por $8 n+1$, com $n \in \mathbb{N}$; sobre o fio $C$ aparecem os (múltiplos de 8)+2, isto é, números que podem ser representados simbolicamente por $8 n+2$, com $n \in \mathbb{N}$ e assim sucessivamente, o que permite organizar os dados conforme a tabela 1. Tabela 1.

\begin{tabular}{|l|l|l|l|l|l|l|l|}
\hline A & B & C & D & E & F & G & H \\
\hline 0 & 1 & 2 & 3 & 4 & 5 & 6 & 7 \\
\hline 8 & 9 & 10 & 11 & 12 & 13 & 14 & 15 \\
\hline 16 & 17 & 18 & 19 & 20 & 21 & 22 & 23 \\
\hline 24 & 25 & 26 & 27 & 28 & 29 & 30 & 31 \\
\hline$\ldots$ & $\ldots$ & $\ldots$ & $\ldots$ & $\ldots$ & $\ldots$ & $\ldots$ & $\ldots$ \\
\hline
\end{tabular}

Obviamente, o problema poderia ter sido resolvido dando continuidade ao processo de construir a tabela até alcançar o número 118, mas essa solução não é prática e ela é inviável para números muito grandes, já que poderíamos perguntar, por exemplo, qual o fio correspondente ao numero 1367? Outro caso em que ela não seria viável é se o problema apresentasse um número de fios muito grande, como por exemplo, uma distribuição com 27 fios.

Assim, para respondermos o questionamento feito, basta verificarmos em qual coluna ou grupo de números se encontra o número 118 e para isso basta sabermos qual é o resto da divisão de 118 por 8 . Podemos verificar facilmente que $118=8 \cdot 14+6$, isto é, o resto da divisão de 118 por 8 é 6, o que significa que 118 pertence ao grupo de números que estão na coluna $\mathrm{G}$ ou sobre o fio $\mathrm{G}$.

Exemplo 3: Prove que $30^{99}+61^{100}$ é divisível por 31 .

Solução do exemplo 3: Observemos que $30 \equiv-1(\bmod 31)$, assim pelo item (vi) da proposição 3 temos

$$
30^{99} \equiv(-1)^{99}(\bmod 31)
$$

e portanto $30^{99} \equiv-1(\bmod 31)$. Analogamente, podemos observar que

$$
61^{99} \equiv(-1)^{100}(\bmod 31)
$$

e portanto $61^{99} \equiv 1(\bmod 31)$. Finalmente, dos resultados acima obtemos,

$$
30^{99}+61^{100} \equiv-1+1(\bmod 31)
$$

Isto é, $30^{99}+61^{100} \equiv 0(\bmod 31)$, o que prova que $30^{99}+61^{100}$ é divisível por 31 .

Exemplo 4: Qual o resto da divisão do número $10^{10}+10^{100}+10^{1000}+\ldots+10^{10000000000}$ por 7 ? Solução do exemplo 4: Como $10 \equiv 3(\bmod 7)$, aplicando o item $(v i)$ da proposição 3 , temos $\operatorname{mas} 3^{10}=\left(3^{5}\right)^{2}=243^{2}$, portanto

$$
10^{10} \equiv 3^{10}(\bmod 7) \text {, }
$$




$$
10 \equiv 243^{2}(\bmod 7) .
$$

Mais ainda, como $243=34 \cdot 7+5$, temos que $243^{2} \equiv 5^{2}(\bmod 7)$ e $5^{2} \equiv 4(\bmod 7)$, obtendo finalmente, pelo item (iii) da proposição 3 , que $10^{10} \equiv 4(\bmod 7)$.

De maneira análoga, podemos mostrar que $10^{100} \equiv 4(\bmod 7), 10^{1000} \equiv 4(\bmod 7)$ e assim sucessivamente até $10^{10000000000} \equiv 4(\bmod 7)$. Podemos concluir então, do item (iv) da proposição 3 que,

$$
10^{10}+10^{100}+10^{1000}+\ldots+10^{10000000000} \equiv 4 \cdot 10(\bmod 7)
$$

e como $40 \equiv 5(\bmod 7)$, obtemos que

$$
10^{10}+10^{100}+10^{1000}+\ldots+10^{10000000000} \equiv 5(\bmod 7)
$$

Aplicação 1: Os trabalhos de Ribeiro (2005) e Pinto (2006) descrevem um dos mecanismos de segurança estabelecido pelo Banco Central Europeu para as notas de Euro que entraram em circulação em 1 de janeiro de 2002, baseado num sistema de congruência módulo 9. O mecanismo é baseado na formação do numero de série, que é composto por uma letra seguida de 11 (onze) algarismos. Segundo Pinto (2006) a letra representa o país do qual é proveniente a nota, os 10 algarismos seguintes servem para numerar a nota, e o ultimo algarismo é o de controle (figura 2).

Tabela 2

\begin{tabular}{lll} 
Letra & País & Valor \\
\hline $\mathrm{L}$ & Finlândia & 4 \\
\hline $\mathrm{M}$ & Portugal & 5 \\
\hline $\mathrm{N}$ & Áustria & 6 \\
\hline $\mathrm{P}$ & Holanda & 8 \\
\hline $\mathrm{R}$ & Luxemburgo & 1 \\
\hline $\mathrm{S}$ & Itália & 2 \\
\hline $\mathrm{T}$ & Irlanda & 3 \\
\hline $\mathrm{U}$ & França & 4 \\
\hline $\mathrm{V}$ & Espanha & 5 \\
\hline $\mathrm{X}$ & Alemanha & 7 \\
\hline $\mathrm{Y}$ & Grécia & 8 \\
\hline $\mathrm{Z}$ & Bélgica & 9
\end{tabular}

Fonte: Pinto, 2006
Figura 2. Nota de Euro

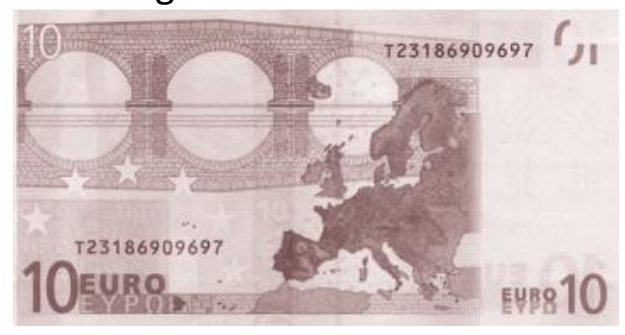

Fonte: Pinto, 2006

O algarismo de controle (C) será um número de 1 a 9 que, somado ao valor numérico (L) proveniente de cada país (tabela 2 ) mais a soma com os próximos 10 algarismos do número de série $\left(x_{1}, x_{2}, \ldots\right)$ dará origem a um número divisível por 9 . Simbolicamente, podemos escrever

$$
L+x_{1}+x_{2}+x_{3}+x_{4}+x_{5}+x_{6}+x_{7}+x_{8}+x_{9}+x_{10}+C \equiv 0(\bmod 9)
$$

O mecanismo de segurança descrito não é eficiente, pelo menos dois erros não são detectados: a troca de dois números (pela propriedade comutativa da adição modular), assim como a troca de um zero por um nove. Ribeiro (2005) critica o sistema adotado já que desde 1990 que o Banco Central Alemão utilizava, nos números de série das suas notas, um sistema que detectava $100 \%$ dos dois principais tipos de erros, então seria esperado que o Banco Central Europeu adotasse, como esquema nos números de série das notas de euro, esse sistema.

Aplicação 2: No Brasil, o Cadastro de Pessoas Físicas junto à Receita Federal (CPF) é composto por 11 dígitos, dividido em dois blocos: um primeiro bloco com 9 algarismos e um segundo bloco com dois, que são os dígitos de controle. Para determinarmos os dígitos de controle, utilizamos também a congruência módulo $m$ (Sá, 2007) . 
Podemos obter o décimo dígito ou primeiro dígito verificador como resultado de uma congruência, módulo 11 de um número obtido por uma operação dos primeiros nove algarismos. Em primeiro lugar atribuímos pesos a cada um dos nove primeiros dígitos: Se $a_{1} a_{2} a_{3} a_{4} a_{5} a_{6} a_{7} a_{8} a_{9}$ é a sequência formada pelos nove primeiros dígitos, devemos multiplicar eles nesta mesma ordem, pela base: $\{1,2,3,4,5,6,7,8,9\}$, e em seguida somar os produtos obtidos. Se denotarmos a soma obtida por $S$, o décimo dígito que denotaremos por $a_{10}$, deverá ser tal que $S-a_{10}$ seja múltiplo de 11 ou $S-a_{10} \equiv 0(\bmod 11)$. Assim, obtemos que $S$ será o próprio resto da divisão por 11 da soma obtida.

Se considerarmos um CPF cujos nove primeiros dígitos são 235343 104, podemos calcular o primeiro dígito de controle seguindo a ideia descrita,

$$
2 \times 1+3 \times 2+5 \times 3+3 \times 4+4 \times 5+3 \times 6+1 \times 7+0 \times 8+4 \times 9=116
$$

Dividindo o resultado obtido por 16 , obtemos $116=11 \times 10+6$, isto é, a divisão por 11 deixa resto 6 , o que mostra que o primeiro dígito de controle é 6 . Para obtermos o segundo dígito de controle, utilizamos o mesmo procedimento acrescentando o décimo dígito que acabamos de calcular e usando uma base de multiplicação de 0 a 9 , assim

$$
2 \times 0+3 \times 1+5 \times 2+3 \times 3+4 \times 4+3 \times 5+1 \times 6+0 \times 7+4 \times 8+6 \times 9=145
$$

Dividindo o resultado obtido por 16 , obtemos $145=11 \times 13+2$, isto é, a divisão por 11 deixa resto 2 , o que mostra que o segundo dígito de controle é 2 . Se o resto da divisão em algum dos dois casos fosse 10, utilizaríamos o dígito zero. Com os resultados obtidos podemos afirmar que o CPF completo é 23534310462 .

Aplicação 3: O ISBN ou International Standard Book Number é um sistema de catalogação de livros, criado em 1967 e oficializado como norma internacional em 1972. Até dezembro de 2006 o ISBN tinha 10 dígitos e a partir de 10 de janeiro de 2007 passaram a ser utilizados 13 dígitos, usaremos as denominações ISBN-10 e ISBN-13 para cada um dos sistemas. Ambos os sistemas, utilizam dígito de controle ou de verificação que pode ser obtido através da aritmética modular. Apresentaremos a forma de calcular o dígito de controle para o sistema ISBN-10, que é o último dos 10 algarismos utilizados.

O sistema ISBN-10 utiliza os nove primeiros dígitos para identificação do livro e os subdivide em três grupos de tamanho variável e separados por hífen. Segundo Colombo (2013), da esquerda para a direita, a primeira parte identifica um grupo nacional ou geográfico de editores, por exemplo, no Brasil esse número é 85 . A segunda parte determina, especificamente, uma editora desse grupo e por fim, a terceira parte, identifica um título específico.

Para entendermos o funcionamento do cálculo do dígito de controle, denotaremos por $a_{1} a_{2} a_{3} a_{4} a_{5} a_{6} a_{7} a_{8} a_{9} X$ a sequência formada pelos dez dígitos, em seguida devemos multiplicar eles nesta mesma ordem, pela base $\{10,9,8,7,6,5,4,3,2,1\}$ somar os produtos obtidos e o resultado deve ser congruente a zero módulo 11 . Isto é, devemos ter,

$$
10 \times a_{1}+9 \times a_{2}+8 \times a_{3}+\ldots+3 \times a_{8}+2 \times a_{9}+X \equiv 0(\bmod 11)
$$

Observando que, se o dígito de controle encontrado for o número 10 , ele será representado pela letra $X$, fazendo referência à numeração romana.

Por exemplo, consideremos o livro Temas e Problemas Elementares, da Coleção Professor de Matemática, da SBM, cujo código do ISBN-10 é 85-85818-29-8. Neste caso, a soma dos produtos será 


$$
\begin{gathered}
S=10 \times 8+9 \times 5+8 \times 8+7 \times 5+6 \times 8+5 \times 1+4 \times 8+3 \times 2+2 \times 9+X= \\
=80+45+64+35+48+5+32+6+18+X
\end{gathered}
$$

Ou seja, $S \equiv 3+1-2+2+4-6-1-5+7+X \equiv 3+X$. Assim, para obtermos $S \equiv 0(\bmod 11)$, devemos ter $3+X \equiv 0(\bmod 11)$ e por isso devemos ter $X=8$.

Consideremos agora o livro Precalculus: Mathematics for Calculus de James Stewart, lothar Redlin e Saleem Watson, publicado em inglês pela editora Thomson Brooks/Cole e cujo código ISBN é dado por 0-534-49277-0. Teremos,

$$
\begin{gathered}
S=10 \times 0+9 \times 5+8 \times 3+7 \times 4+6 \times 4+5 \times 9+4 \times 2+3 \times 7+2 \times 7+X= \\
=0+45+24+28+24+45+8+21+14+X
\end{gathered}
$$

Ou seja, $S \equiv 0+1+2-5+2+1-3-1+3+X \equiv 0+X$. Assim, para obtermos $S \equiv 0(\bmod 11)$, devemos ter $X \equiv 0(\bmod 11)$ e por isso devemos ter $X=0$.

\section{DISCUSSÃO}

Através da resolução de exercícios e seminários apresentados durante o desenvolvimento do trabalho foi possível verificar a vasta aplicação dos conceitos de congruência e divisibilidade tanto em disciplinas de ensino regular, quanto relacionados ao cotidiano. Foram analisadas aplicações em resoluções de exercícios da Olimpíada Brasileira de Matemática e também aplicações tais como, determinação de códigos de segurança (CPF, ISBN) e também um dos mecanismos de segurança das primeiras notas de Euro lançadas em 2002.

\section{CONCLUSÃO}

Através do presente trabalho foi possível verificar a utilidade dos conceitos de congruência e divisibilidade tanto para resolver problemas matemáticos, quanto para solucionar problemas que podem ser encontrados no cotidiano.

\section{REFERÊNCIAS}

Colombo, J.P.E. Aritmética: Códigos de Barras e outras Aplicações de Congruências. 2013. 63 f. Dissertação (Mestrado Profissional em Matemática em Rede nacional - PROFMAT). Universidade Federal de Mato Grosso do Sul. Campo Grande - MS.

OBMEP: Banco de Questões. Rio de Janeiro: IMPA, 2010. Disponível em: <http://www.obmep.org.br >. Consultado em: 05/08/2016.

Pinto, H. Sistemas de Identificação com Algarismos de Controlo. Educação e Matemática No. 86 (Janeiro/Fevereiro). p. 19 a 21. 2006.

Ribeiro, C.M. A Álgebra dos Sistemas de Identificação. Ata do Encontro da Sociedade Portuguesa de Investigação em Educação Matemática: Números e Álgebra na aprendizagem da Matemática e na Formação de Professores. Caminha, 2005. Disponível em: <http://spiem.pt/publicacoes/arquivo/encontro-2005/>. Consultado em: 12/08/2016.

Sá, I.P. Aritmética Modular e algumas de suas Aplicações. Minicurso. IX ENEM (Encontro Nacional de Educação Matemática). 18 a 21 de julho de 2007. Disponível em: <http://www.magiadamatematica.com/>. Consultado em: 05/08/2016

Agência Brasileira do ISBN. Informe ISBN 13 Dígitos. Disponível em: <http://www.isbn.bn.br/website/>. Consultado em: 12/08/2016 